\title{
RATE-BASED VERSUS DISTORTION-BASED OPTIMAL JOINT SOURCE-CHANNEL CODING
}

\author{
Raouf Hamzaoui, Vladimir Stanković \\ Institut für Informatik, Universität Leipzig \\ Augustusplatz 10-11, 04109 Leipzig \\ hamzaoui,stankovi@informatik.uni-leipzig.de
}

\author{
Zixiang Xiong \\ Texas A \&M University \\ College Station, TX 77843 \\ zx@lena.tamu.edu
}

\begin{abstract}
We consider a joint source-channel coding system that protects an embedded wavelet bitstream against noise using a finite family of channel codes with error detection and error correction capability. The performance of this system may be measured by the expected distortion or by the expected number of correctly received source bits subject to a target total transmission rate. Whereas a rate-based optimal solution can be found in linear time, the computation of a distortion-based optimal solution is prohibitive. Under the assumption of the convexity of the operational distortion-rate function of the source coder, we give a lower bound on the expected distortion of a distortion-based optimal solution that depends only on a rate-based optimal solution. Then we show that a distortion-based optimal solution provides a stronger error protection than a rate-based optimal solution and exploit this result to reduce the time complexity of the distortion-based optimization. Finally, we propose a fast iterative improvement algorithm that starts from a rate-based optimal solution and converges to a local minimum of the expected distortion. Experimental results for a binary symmetric channel with the SPIHT coder and JPEG 2000 show that our lower bound is close to optimal. Moreover, the solution given by our local search algorithm has about the same quality as a distortion-based optimal solution, whereas its complexity is much lower than that of the previous best solution.
\end{abstract}

\section{INTRODUCTION}

One of the most successful systems for the robust progressive transmission of embedded wavelet image codes over memoryless noisy channels was introduced by Sherwood and Zeger [1]. The basic idea is to protect the source bitstream, for instance the SPIHT code [2], with a concatenation of an outer cyclic redundancy-check (CRC) coder and an inner rate-compatible punctured convolutional (RCPC) coder and to stop the decoding when the first error is detected. This system was also efficiently used by Banister, Belzer, and Fischer ([3]) for protecting JPEG 2000 [4] coded images (RCPC codes were replaced by punctured turbo codes) and by Xiong, Kim, and Pearlman [5] for video transmission. In the original setting $[1,6]$, the information bits were organized in packets of fixed-length, each of which was protected by appending a variable number of extra bits. However, it is more convenient to allow the blocks of information bits to have a variable length and to fix the size of the channel codewords $[3,7]$.

A challenging problem is to find an allocation of the transmitted bits between the source and the channel that minimizes the expected distortion subject to a target total bit rate. For the fixed source packet length case, Lu, Nosratinia, and Aazhang [8] show that under some 
assumptions, the channel code rates of an optimal solution should be nondecreasing with the packet number, which significantly reduces the complexity of an exhaustive search. Also in the fixed source packet length case, Chande and Farvardin [6] provide a dynamic programming solution to the optimization problem and report an $O\left(R^{2}\right)$ time complexity, where $R$ is the target total transmission rate. In the fixed channel codeword length case, however, no fast exact solution is known. To the best of our knowledge, the best approximation to an optimal solution was proposed in [3]. It is based on a Viterbi algorithm and has $O\left(R^{2}\right)$ complexity. However, this result holds only for particular sets of channel code rates. For an arbitrary channel coder, the worst case complexity is exponential in the total transmission rate.

An alternative to minimizing the expected distortion is to maximize the expected number of correctly received source bits. Though suboptimal in the distortion sense, this ratebased approach has two main advantages: an optimal solution can be computed with a linear-time algorithm $[6,7]$, and it is independent of both the image and the source coder performance; thus, it can be determined by the receiver, which avoids the need for side information. In [9], experimental results show that the solutions to the two optimization problems have a similar performance for the SPIHT coder and an RCPC channel coder (the loss in peak-signal-to-noise ratio (PSNR) was less than $0.2 \mathrm{~dB}$ for the $512 \times 512$ gray-scale Lenna image). Hedayat and Nosratinia [10] analytically confirm these results under many assumptions, including an i.i.d. Gaussian source and a perfect progressive source coder that achieves the rate-distortion function.

In this paper, we answer the following important questions: Is it possible to theoretically estimate the loss in reconstruction quality when we replace the minimization of the expected distortion by the maximization of the expected number of correctly received source bits? Can one decrease the time complexity of the distortion-based minimization? Finally, can one rapidly find a better solution than the one obtained by the rate-based maximization?

The paper is organized as follows. In Section 2, we set up our notations and state the two optimization problems in the context of fixed-length channel codewords. In Section 3, we provide a theoretical upper bound on the difference in expected distortion between the solutions of the two optimization problems under the assumption of the convexity of the operational distortion-rate function of the source coder. We also show that the number of source bits included in the packets of a distortion-based optimal solution is smaller than that of a rate-based optimal solution. Then we use this result to reduce the complexity of the distortion-based minimization by pruning the set of candidates. In Section 4, we propose a fast local search algorithm that starts from a rate-based optimal solution and iteratively tries to reduce the expected distortion by modifying at each step the protection of one packet. In Section 5, we present numerical results for the SPIHT and JPEG 2000 coders. We show in particular that the local search algorithm yields comparable solutions to that obtained with the Viterbi algorithm of [3], but at a much lower complexity. In the last section, we discuss our results and suggest future work.

\section{OPTIMIZATION CRITERIA}

We consider a system that protects an embedded source code using a finite family of channel codes with error detection and error correction capability. The channel encoder 
transforms the information bitstream into a sequence of channel codewords (packets) of fixed length $L$. If the decoder detects an error, then the transmission is stopped, and the image is reconstructed from the correctly received source bits. We assume that all errors can be detected. Given $m$ channel codes $c_{1}, \ldots, c_{m}$, let $\mathcal{R}$ be the set of corresponding code rates $r_{1}<r_{2}<\cdots<r_{m}$. For $i=1, \ldots, m$, let $p\left(r_{i}\right)$ denote the probability of a decoding error in a packet protected by code $c_{i}$. We may assume without loss of generality that $p\left(r_{1}\right)<\cdots<p\left(r_{m}\right)<1$. Suppose now that we want to send $N$ packets of $L$ bits each. Then an $N$-packet error protection scheme (EPS) $R=\left(r_{k_{1}}, \ldots, r_{k_{N}}\right)$ assigns to each packet $i, i=1, \ldots, N$, a channel code rate $r_{k_{i}} \in \mathcal{R}$. For $i=1, \ldots, N-1$, $P_{i}(R)=\prod_{j=1}^{i}\left(1-p\left(r_{k_{j}}\right)\right) p\left(r_{k_{i+1}}\right)$ is the probability that no errors occur in the first $i$ packets with an error in the next one, $P_{0}(R)=p\left(r_{k_{1}}\right)$ is the probability of an error in the first packet, and $P_{N}(R)=\prod_{j=1}^{N}\left(1-p\left(r_{k_{j}}\right)\right)$ is the probability that all $N$ packets are correctly received. For an $N$-packet EPS $R=\left(r_{k_{1}}, \ldots, r_{k_{N}}\right) \in \mathcal{R}^{N}$, the expected distortion is given by

$$
E_{N}[d]\left(r_{k_{1}}, \ldots, r_{k_{N}}\right)=\sum_{i=0}^{N} P_{i}(R) d_{i}(R),
$$

where $d_{0}(R)=d_{0}$ is a constant, and for $i \geq 1, d_{i}(R)$ is the reconstruction error using the first $i$ packets. Since the number of possible $N$-packet EPS's is equal to $m^{N}$, brute force cannot be used to minimize (1) when $N$ is large. However, if we replace the minimization of (1) by the maximization of the expected number of correctly received source bits

$$
E_{N}[r]\left(r_{k_{1}}, \ldots, r_{k_{N}}\right)=\sum_{i=0}^{N} P_{i}(R) V_{i}(R),
$$

where $V_{0}(R)=0$ and for $i \geq 1, V_{i}(R)=\sum_{j=1}^{i} v\left(r_{k_{j}}\right)$ with $v\left(r_{k_{j}}\right)=L r_{k_{j}}$ being the number of source bits in the $j$ th packet, then an optimal solution can be computed in $O(N)$ time [7]. Maximizing (2) is reasonable for an efficient embedded coder because we expect the average distortion to decrease when the average number of correctly received source bits increases. Note, however, that the two optimizations do not necessarily yield the same EPS (see Section 5). In the following, we say that an EPS that minimizes (1) is distortion optimal and that an EPS that maximizes (2) is rate optimal. In [3], a Viterbi algorithm is used to find an approximation to a distortion-optimal EPS. However, the reported $O\left(N^{2}\right)$ time complexity is valid only for some particular sets of channel code rates. In many important cases, the number of nodes in each stage of the Viterbi trellis does not grow linearly, and the complexity of the algorithm is exponential in the number of packets $N$.

Whereas the channel code rates used by a rate-optimal solution are nondecreasing with the packet number [7], this is not necessarily true for a distortion-optimal solution as shown by the following counter-example. Suppose that we have two packets $(N=2)$ and two channel code rates $r_{1}<r_{2}(m=2)$. Then $\left(r_{1}, r_{1}\right),\left(r_{1}, r_{2}\right),\left(r_{2}, r_{1}\right)$, and $\left(r_{2}, r_{2}\right)$ are the four possible 2-packet EPS's. Suppose now that $p\left(r_{1}\right)=0.09$ and $p\left(r_{2}\right)=0.1$. Let $d_{0}=100, d_{1}\left(r_{1}, r_{1}\right)=d_{1}\left(r_{1}, r_{2}\right)=95, d_{1}\left(r_{2}, r_{1}\right)=d_{1}\left(r_{2}, r_{2}\right)=50, d_{2}\left(r_{1}, r_{1}\right)=$ $20, d_{2}\left(r_{1}, r_{2}\right)=d_{2}\left(r_{2}, r_{1}\right)=0.001$, and $d_{2}\left(r_{2}, r_{2}\right)=0.0005$. Then it is easy to check that $E_{N}[d]\left(r_{2}, r_{1}\right)<E_{N}[d]\left(r_{2}, r_{2}\right)<E_{N}[d]\left(r_{1}, r_{2}\right)<E_{N}[d]\left(r_{1}, r_{1}\right)$. Thus, the EPS $\left(r_{2}, r_{1}\right)$ is distortion optimal. An $N$-packet EPS that minimizes (1) under the constraint 
$r_{k_{1}} \leq \cdots \leq r_{k_{N}}$ will be called constrained distortion optimal. In the above example, $\left(r_{2}, r_{2}\right)$ is a constrained distortion-optimal EPS. Note that the constrained minimization reduces the number of candidates from $m^{N}$ to $\left(\begin{array}{c}m+N-1 \\ N\end{array}\right)$.

\section{ERROR BOUND}

Because the number of $N$-packet EPS's is too high, we do not know the approximation error made if we use a rate-optimal solution instead of a distortion-optimal one. However, if we assume that the operational distortion-rate function of the source coder is nonincreasing and convex, then we can compute an upper bound for this error as follows.

Proposition 1 Let $f$ be the operational distortion-rate function of the source coder. Suppose that $f$ is nonincreasing and convex. Let $T^{*}$ be a distortion-optimal $N$-packet EPS and let $R^{*}$ be a rate-optimal $N$-packet EPS. Then we have

(i) $E_{N}[d]\left(T^{*}\right) \geq f\left(E_{N}[r]\left(R^{*}\right)\right)$.

(ii) $V_{N}\left(T^{*}\right) \leq V_{N}\left(R^{*}\right)$ and the inequality is strict if $T^{*}$ is not rate optimal.

Proof. Let $R$ be an $N$-packet EPS. Because $\sum_{i=0}^{N} P_{i}(R)=1$, we know that $E_{N}[d](R)$ (respectively $E_{N}[r](R)$ ) is contained in the interval $\left[d_{N}(R), d_{0}\right]$ (respectively $\left[0, V_{N}(R)\right]$ ).

(i) Let $M_{0}$ and $M_{N}(R)$ denote the points on the distortion-rate curve whose coordinates are $\left(0, d_{0}\right)$ and $\left(V_{N}(R), d_{N}(R)\right)$, respectively. Since $f$ is convex, the chord $\left[M_{0} M_{N}(R)\right]$ is above the $\operatorname{arc}\left(M_{0} M_{N}(R)\right)$. Thus, $E_{N}[r](R) \leq E_{N}[r]\left(R^{*}\right)$ gives the inequality $E_{N}[d](R) \geq$ $f\left(E_{N}[r](R)\right) \geq f\left(E_{N}[r]\left(R^{*}\right)\right)$.

(ii) Suppose that $V_{N}(R)>V_{N}\left(R^{*}\right)$. Let $M_{N}\left(R^{*}\right)$ denote the point on the distortion-rate curve whose coordinates are $\left(V_{N}\left(R^{*}\right), d_{N}\left(R^{*}\right)\right)$. Since $E_{N}[r](R) \leq E_{N}[r]\left(R^{*}\right)$ and the slope of the chord $\left[M_{0} M_{N}\left(R^{*}\right)\right]$ is strictly smaller than the slope of the chord $\left[M_{0} M_{N}(R)\right]$, we obtain $E_{N}[d](R)>E_{N}[d]\left(R^{*}\right)$. Thus, $R$ cannot be distortion optimal. This proves that $V_{N}\left(T^{*}\right) \leq V_{N}\left(R^{*}\right)$. Moreover, a similar slope argument shows that if $R$ is not rate optimal and $V_{N}(R)=V_{N}\left(R^{*}\right)$, then $R$ cannot be distortion optimal, which completes the proof.

Note that the lower bound on the optimal expected distortion given in (i) can be efficiently computed because the algorithm of [7] finds a rate-optimal EPS in $O(N)$ time. On the other hand, Proposition 1 (ii) allows us to reduce the complexity of the distortion-based optimization by discarding many schemes. We first introduce a definition.

Definition 1 An $N$-packet EPS $R$ provides a stronger protection than an $N$-packet EPS $S$ if $V_{N}(R)<V_{N}(S)$.

Given an $N$-packet EPS $R$, one can consider the finite set $A_{R}=\left\{R_{1}, \ldots, R_{n_{R}}\right\} \subset \mathcal{R}^{N}$ of all $N$-packet EPS's that provide a stronger protection than $R$ and such that for $i \geq$ $1, V_{N}\left(R_{i+1}\right) \leq V_{N}\left(R_{i}\right)$. For example, suppose that $\mathcal{R}=\left\{r_{1}, r_{2}\right\}$. Let $R=\left(r_{1}, r_{2}, r_{2}\right)$. Then $A_{R}$ consists of the four schemes $R_{1}=\left(r_{1}, r_{2}, r_{1}\right), R_{2}=\left(r_{1}, r_{1}, r_{2}\right), R_{3}=\left(r_{2}, r_{1}, r_{1}\right)$, and $R_{4}=\left(r_{1}, r_{1}, r_{1}\right)$. We now have

Proposition 2 Suppose that the distortion-rate function of the source coder is nonincreasing and convex. Then the following algorithm finds a distortion-optimal N-packet EPS T* 1. Set $i=1$. Use the algorithm of [7] to compute a rate-optimal $N$-packet EPS $R^{*}$. Let 
$n_{R^{*}}$ be the number of elements in $A_{R^{*}}$. Set $T^{*}=R^{*}$.

2. Let $R_{i}$ be the ith EPS in $A_{R^{*}}$. If $E_{N}[d]\left(R_{i}\right)<E_{N}[d]\left(T^{*}\right)$, set $T^{*}=R_{i}$.

3. If $d_{N}\left(R_{i}\right)>E_{N}[d]\left(T^{*}\right)$, stop.

4. Set $i=i+1$. If $i>n_{R^{*}}$, stop. Otherwise, go to Step 2 .

\section{LOCAL SEARCH ALGORITHM}

The algorithm of Proposition 2 significantly accelerates the computation of a constrained distortion-optimal EPS. However, the computation time may be unacceptable for some applications where speed is a priority. In this section, we propose a local search algorithm that rapidly finds a local minimum of (1). Experimental results in Section 5 show that this local minimum is near a global one. We first define the neighborhood of a solution.

Definition 2 Let $\mathcal{R}$ be a set of code rates and let $R \in \mathcal{R}^{N}$ be an N-packet EPS with nondecreasing rates. Then the neighborhood of $R$ is the set of EPS's $S \in \mathcal{R}^{N}$ such that

(a) $V_{N}(S)<V_{N}(R)$.

(b) $R$ and $S$ differ in only one code rate.

(c) The rates of $S$ are nondecreasing.

We now need to precise an indexing of the solutions contained in a neighborhood. We define the $k$ th neighbor of $R$ as the one whose new code rate (according to (b) in Definition 2) is the $k$ th largest one. For example, let $m=4$ and $R=\left(r_{1}, r_{3}, r_{3}, r_{4}\right)$ be a four-packet EPS. Then the neighborhood of $R$ consists of the set of schemes $\left\{\left(r_{1}, r_{2}, r_{3}, r_{4}\right),\left(r_{1}, r_{1}, r_{3}, r_{4}\right)\right.$, $\left.\left(r_{1}, r_{3}, r_{3}, r_{3}\right)\right\}$ which are ordered as follows: $\left(r_{1}, r_{3}, r_{3}, r_{3}\right),\left(r_{1}, r_{2}, r_{3}, r_{4}\right),\left(r_{1}, r_{1}, r_{3}, r_{4}\right)$.

The local search algorithm works by iterative improvement. We start from a rate-optimal solution. Then we consider the first neighbor of the current solution. If the expected distortion of this neighbor is smaller than that of the current solution, then we update the current solution; otherwise we consider the next neighbor and repeat the procedure. Note that the neighbors of a given EPS $R$ provide a stronger protection than $R$. This is in accordance with Proposition 1 (ii). A pseudo-code for the local search algorithm is given below.

\section{Local search algorithm}

1. Set $k=1, l=1$, and $n=0$. Use the algorithm of [7] to compute a rate-optimal $N$-packet EPS $R_{n}$.

2. Let $r$ be the $k$ th highest rate used by $R_{n}$. Let $j$ be the index of the first packet that $R_{n}$ protects with $r$. If $r=r_{1}$, stop. Otherwise, let $r_{c} \in \mathcal{R}$ be the $l$ th highest rate smaller than $r$ and define $R_{c}$ to be the EPS obtained from $R_{n}$ by protecting packet $j$ with $r_{c}$.

3. If $E_{N}[d]\left(R_{c}\right)<E_{N}[d]\left(R_{n}\right)$, set $R_{n+1}=R_{c}, n=n+1, k=1, l=1$, and go to Step 2 . 4. If $j \neq 1$ and $r_{c}$ is greater than the rate of packet $j-1$, set $l=l+1$. If $j \neq 1$ and $r_{c}$ is equal to the rate of packet $j-1$, set $l=1$ and $k=k+1$. If $j=1$ and $r_{c} \neq r_{1}$, set $l=l+1$. If $j=1$ and $r_{c}=r_{1}$, stop.

5. Go to Step 2.

Simulations show that the above local search algorithm can be slightly improved by removing $k=1, l=1$ from Step 3. We use this variant in our experimental results. 


\section{RESULTS}

In this section, we numerically compare the mean-squared error (MSE) performance and the time complexity of a rate-optimal solution, a constrained MSE-optimal solution, the solution computed by the Viterbi algorithm of [3], and the solution of the local search algorithm. The test image was the standard 8 bits per pixel (bpp) $512 \times 512$ gray scale Lenna. Results are given for Fowler's implementation of the SPIHT coder [11] and for a Java implementation of JPEG 2000 [12]. Note that the operational distortion-rate function $d=f(r)$ (where $d$ is the MSE) of the SPIHT coder can be modeled as a convex function [13]. We recall that $N$ and $L$ denote the target number of packets and the length of a channel codeword, respectively. Thus, the total transmission rate was $R_{T}=N L / n^{2}$ bpp for $n \times n$ images. The packets were sent over a binary symmetric channel and protected with a concatenation of a CRC-32 coder and a rate compatible punctured turbo coder [14]. The generator polynomial of the CRC code was $(32,26,23,22,16,12,11,10,8,7,5,4,2,1,0)$. The turbo coder consisted of two identical recursive systematic convolutional encoders with memory length 4 and generators $(31,27$ ) (octal). The mother code was $20 / 60=1 / 3$, and the puncturing rate was 20 , yielding 41 possible channel code rates. The length of a packet was equal to $L=2048$ bits, consisting of a variable number of source bits, 32 CRC bits, 4 bits to set the turbo encoder into a state of all zeroes, and protection bits. We used iterative maximum a posteriori decoding, which was stopped if no correct sequence was found after 20 iterations. The bit error rate of the binary symmetric channel was 0.1 . The probability of a packet decoding error for each code rate was computed with 50000 Monte Carlo simulations. Only the channel code rates that may be selected by a rate-optimal EPS were considered in MSE-minimization. Thus, $m=4$ with $r_{1}=\frac{20}{56}, r_{2}=\frac{20}{52}, r_{3}=\frac{20}{50}$, and $r_{4}=\frac{20}{48}$ [7]. The corresponding numbers of source bits per packet were $v\left(r_{1}\right)=$ $695, v\left(r_{2}\right)=751, v\left(r_{3}\right)=783$, and $v\left(r_{4}\right)=817$. The probabilities of packet decoding failure were $p\left(r_{1}\right)=0.00001, p\left(r_{2}\right)=0.0002, p\left(r_{3}\right)=0.00117$, and $p\left(r_{4}\right)=0.00449$.

Figure 1 shows the bound predicted by Proposition 1, the expected MSE performance of a constrained MSE-optimal solution and that of a rate-optimal solution. We point out that we did not consider the side information that must be sent to the decoder to specify an MSEoptimal solution. Because the difference between the lower bound and the performance of a constrained MSE-optimal solution is very small, we conclude that a constrained MSEoptimal solution is near MSE-optimal. For the two coders the rate-optimal solution was a good approximation to the constrained MSE-optimal solution. However, the approximation error was larger for JPEG 2000.

Figure 2 (a) shows for the SPIHT coder the difference in expected MSE between a solution found by rate optimization and a constrained MSE-optimal solution, and the difference between a solution found by the local search algorithm and a constrained MSEoptimal solution. Figure 2 (b) shows the difference in expected MSE between respectively a rate-optimal solution, a local search solution, a constrained MSE-optimal solution and the bound of Proposition 1. The results show that except for one transmission rate where the initial rate-optimal solution was poor, the solution found by local search was very close to optimal.

Figure 3 presents similar results for JPEG 2000. Here, for clarity of display, we give the difference in PSNR of the expected MSE. That is, for two solutions $R_{1}$ and $R_{2}$, the figure 


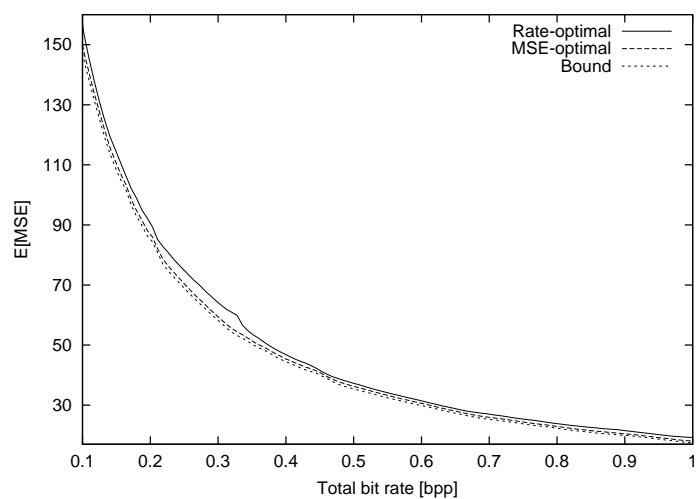

(a)

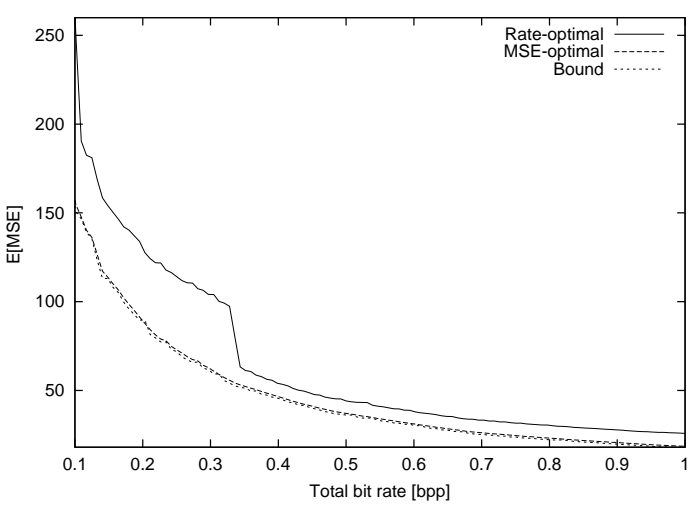

(b)

Figure 1: Bound of Proposition 1, expected MSE of a constrained MSE-optimal solution, and expected MSE of a rate-optimal solution for the SPIHT coder (a) and JPEG 2000 (b).

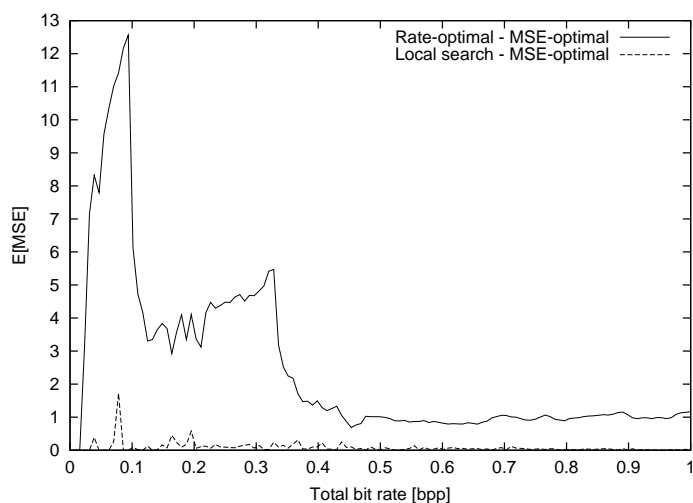

(a)

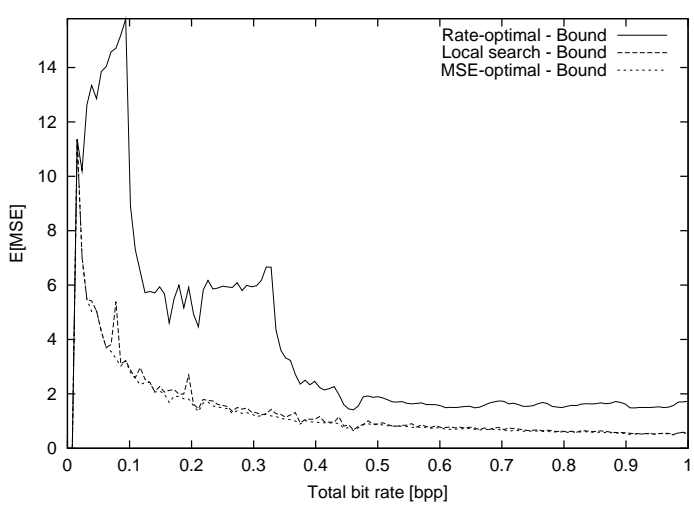

(b)

Figure 2: SPIHT coder. (a) Difference in expected MSE between, respectively, a rateoptimal solution, a local search solution and a constrained MSE-optimal solution. (b) Difference in expected MSE between, respectively, a rate-optimal solution, a local search solution, a constrained MSE-optimal solution and the bound of Proposition 1.

shows the difference

$$
10 \log _{10} \frac{255^{2}}{E_{N}[d]\left(R_{1}\right)}-10 \log _{10} \frac{255^{2}}{E_{N}[d]\left(R_{2}\right)} .
$$

In the above experiments, the constrained MSE-optimal solution was computed with a brute-force algorithm. However, one can speed-up this minimization by using the algorithm of Proposition 2 with the same monotonicity constraint. For the SPIHT coder, our algorithm found the same solution as the brute force algorithm. For JPEG 2000, the expected MSE of the two solutions was identical up to the second decimal. This indicates that the convexity assumption was acceptable for both coders.

Finally, Table 1 compares for the SPIHT coder the CPU time and the expected MSE of, respectively, a rate-optimal solution computed with the algorithm in [7], a constrained MSE-optimal solution computed with the algorithm of Proposition 2, a solution found by 


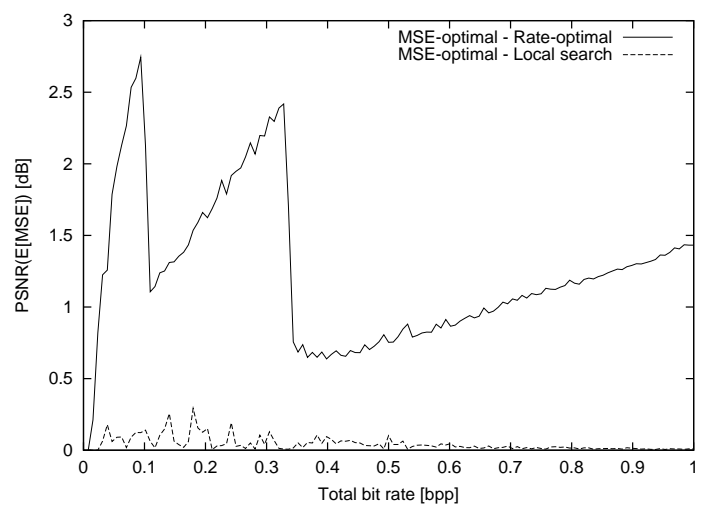

(a)

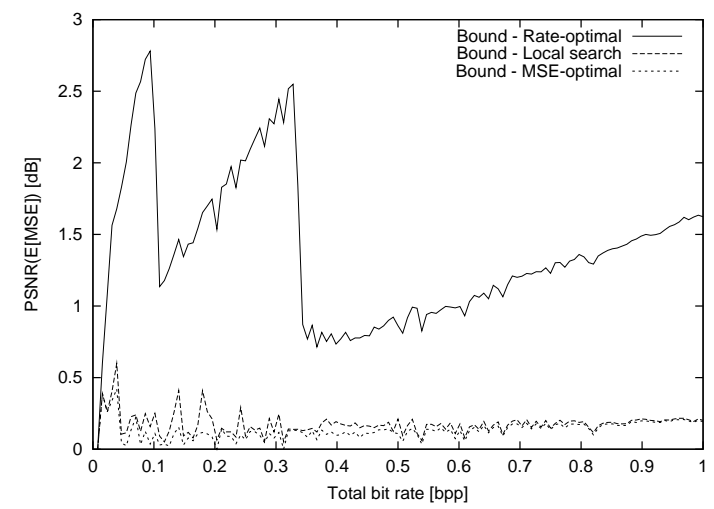

(b)

Figure 3: JPEG 2000. (a) Difference in PSNR of the expected MSE between a constrained MSE-optimal solution and a rate-optimal solution, and a local search solution, respectively. (b) Difference in PSNR of the expected MSE between the bound of Proposition 1 and a rate-optimal solution, a local search solution, and a constrained MSE-optimal solution, respectively.

the Viterbi algorithm of [3] (this algorithm also uses the monotonicity constraint on the channel code rates), and a solution computed with our local search algorithm. The CPU time was measured on an SGI O2 having four $270 \mathrm{MHz}$ MIPS R12000 processors and a main memory size of 1536 Megabytes. The local search algorithm found a solution with about the same quality as the constrained distortion-optimal solution. Moreover, the time complexity of local search was much lower than that of the Viterbi algorithm. We obtained similar results for JPEG 2000.

\begin{tabular}{ccccccccc}
\hline Total rate & \multicolumn{2}{c}{ CDO } & \multicolumn{2}{c}{ RO } & \multicolumn{2}{c}{ LS } & \multicolumn{2}{c}{ Viterbi } \\
(bpp) & MSE & Time (s) & MSE & Time (s) & MSE & Time (s) & MSE & Time (s) \\
\hline 0.25 & 70.42 & 2.39 & 74.90 & $<0.01$ & 70.52 & 0.2 & 70.42 & 6.85 \\
0.5 & 36.27 & 40.9 & 37.28 & $<0.01$ & 36.28 & 0.22 & 36.34 & 72.42 \\
0.75 & 24.40 & 189.64 & 25.34 & $<0.01$ & 24.44 & 0.23 & 24.53 & 288.57 \\
1.0 & 18.02 & 577.9 & 19.20 & $<0.01$ & 18.03 & 0.3 & 18.13 & 777.68 \\
\hline
\end{tabular}

Table 1: Expected MSE and CPU time in seconds at various transmission rates for a constrained MSE-optimal solution (CDO), a rate-optimal solution (RO), a solution found by the local search algorithm (LS), and one obtained with the Viterbi algorithm [3].

\section{CONCLUSION}

For the original system of Sherwood and Zeger [1], in which the packets of information bits have a fixed length, a distortion-optimal EPS can be computed with dynamic programming in $O\left(R^{2}\right)$ time where $R$ is the transmission rate [6,9]. However, in the fixed channel codeword length setting, there is no algorithm that computes a distortion-optimal EPS in 
reasonable time. The best previous approximate solution is based on a Viterbi algorithm [3].

We showed that for a source coder whose operational distortion-rate function is nonincreasing and convex, a sharp lower bound on the performance of a distortion-optimal solution can be quickly computed. This lower bound is useful to evaluate the quality of approximate solutions. We also proved that a distortion-optimal solution is either rate optimal or provides a stronger protection than a rate-optimal solution. Then, we proposed a local search algorithm that starts from a rate-optimal solution and converges to a locally distortion-optimal solution. Experimental results with the SPIHT coder and JPEG 2000 showed that both the local search algorithm and the Viterbi algorithm found comparable high-quality solutions; however, the local search algorithm was much faster.

In this paper, our goal was to minimize the expected distortion. If we prefer instead to maximize the expected PSNR, then, by assuming that the operational PSNR-rate function is nondecreasing and concave, we can use results similar to those in Propositions 1 and 2.

Finally, we point out that Proposition 2 can be used to speed-up the $O\left(R^{2}\right)$ dynamic programming algorithm of $[6,9]$ in the context of fixed-length information packets.

Acknowledgments. We thank Michael Hiller for implementing the Viterbi algorithm of [3] and Brian Banister for providing us with the brute-force algorithm that computes a constrained distortion-optimal solution.

\section{References}

[1] P.G. Sherwood and K. Zeger, "Progressive image coding on noisy channels," Proc. DCC’97 Data Compression Conference, pp. 72-79, Snowbird, Utah, March 1997.

[2] A. Said and W. A. Pearlman, "A new fast and efficient image codec based on set partitioning in hierarchical trees," IEEE Trans. Circuits and Systems for Video Technology, Vol. 6, pp. 243-250, June 1996.

[3] B.A. Banister, B. Belzer, and T.R. Fischer, "Robust image transmission using JPEG2000 and Turbo-codes," Proc. IEEE ICIP-2000, vol 1, pp. 371-374, Vancouver, Oct. 2000.

[4] D. Taubman and M. Marcellin, JPEG2000: Image Compression Fundamentals, Standards, and Practice, Kluwer, 2001.

[5] Z. Xiong, B. Kim, and W. Pearlman, "Progressive video coding for noisy channels," Proc. IEEE ICIP-1998, Chicago, Illinois, Oct. 1998.

[6] V. Chande and N. Farvardin, "Joint source-channel coding for progressive transmission of embedded source coders," Proc. DCC'99 Data Compression Conference, pp. 52-61, Snowbird, Utah, March 1999.

[7] V. Stanković, R. Hamzaoui, and D. Saupe, "Fast algorithm for optimal error protection of embedded wavelet codes," Proc. MMSP-01 IEEE Workshop on Multimedia Signal Processing, pp. 593-598, Cannes, Oct. 2001. 
[8] J. Lu, A. Nosratinia, and B. Aazhang, "Progressive source-channel coding of images over bursty error channels," Proc. IEEE ICIP-1998, pp. 127-131, Chicago, Illinois, Oct. 1998.

[9] V. Chande and N. Farvardin, "Progressive transmission of images over memoryless channels," IEEE Journal on Selected Areas in Communications, vol. 18, number 6, pp. 850-860, June 2000.

[10] A. Hedayat and A. Nosratinia, "Rate allocation in source-channel coding of images," Proc. IEEE ICIP-2001, vol. 1, pp. 189-192, Thessaloniki, Oct. 2001.

[11] J.E. Fowler, "An open source software library for quantization, compression and coding," Applications of Digital Image Processing XXIII, Proc. SPIE 4115, pp. 294-301, August 2000.

[12] M. Charrier, D. Santa Cruz, and M. Larsson, "JPEG2000, the next millennium compression standard for still images," Proc. IEEE ICMCS'99, 1(2), pp. 131-132, Florence, June 1999.

[13] A. Appadwedula, D. L. Jones, K. Ramchandran, and I. Konzintsev, "Joint source channel matching for a wireless communications link," Proc. IEEE ICC'98, Atlanta, Georgia, pp. 482-486, June 1998.

[14] D.N. Rowitch and L.B. Milstein, "Rate compatible punctured turbo (RCPT) codes in a hybrid FEC/ARQ System,” Proc. Globecom'97, vol. 4., pp. 55-59, Phoenix, AZ, Nov. 1997. 